Staphylococcus aureus in Ontario. Can Commun Dis Rep 1997;23:45-46.

4. Chambers HF. Methicillin-resistant staphylococci. Clin Microbiol Rev 1988;1:173-186.

5. Vannuffel P, Gigi J, Ezzedine H, Vandercam B, Delmee M, Wauters G, et al. Specific detection of methicillin-resistant Staphylococcus species by multiplex PCR / Clin Microbiol 1995;33:2864-2867.

6. Brakstad OG, Aasbakk K, Maeland JA. Detection of Staphylococcus aureus by polymerase chain reaction amplification of $n u c$ gene. J Clin Microbiol 1992;30:1654-1660.

7. Cavassini $M$, Wenger A, Jaton $K$, Blanc DS, Bille J. Evaluation of MRSA-Screen, a simple anti-PBP 2a slide latex agglutination kit, for rapid detection of methicillin resistance in Staphylococcus aureus. I Clin Microbiol 1999;37:1591-1594.

Zafar Hussain, MD

Michael A. John, MD

London Health Sciences Centre London, Ontario, Canada

\section{Déjà Vu ... All Over Again? The Importance of Instrument Drying}

\section{To the Editor:}

Although the risk of patient infection following flexible endoscopy is reported to be very low, investigations linking nosocomial infection (and pseudo-infection) to endoscopes contaminated with waterborne microorganisms have been published., ${ }^{1,2}$ In 1991, a report documenting patient infection and pseudo-infection caused by two models of automated flexible endoscope reprocessors (AFERs), whose internal components were colonized with Pseudomonas aeruginosa and Mycobacterium chelonae, was published in Morbidity and Mortality Weekly Report (MMWR). ${ }^{3}$ Discussed in this report (and several others) is the importance of drying the endoscope to prevent bacterial colonization during storage. ${ }^{1-3}$ Drying is typically achieved by rinsing each of the endoscope's channels with $70 \%$ alcohol (to facilitate drying), followed by forced air. ${ }^{1-3}$

More recently, an outbreak linked to endoscopes contaminated with $P$ aeruginosa was reported in $M M W R^{4}$ Investigators of this outbreak, referred to as cluster 3 , concluded that inadequately trained healthcare staff improperly connected bronchoscopes to an AFER, resulting in multiple patient infections and one fatality (for unclear reasons, the fatality was not reported in this $M M W R$ ).

Although several noteworthy recommendations were provided in the $M M W R,{ }^{4}$ absent was a needed discussion of the importance of drying the endoscope to prevent patient infection. Also not discussed in this $M M W R^{4}$ was whether the AFER's filtered rinse water and the hospital's water supply, water faucets, and sink drains were sampled microbiologically.

Obtaining cultures of these sites, as well as of the AFER's internal components and water filters, has been recommended during an investigation to identify the source of, and risk factors for, patient infection. ${ }^{1,2}$ Indeed, these sampling data are crucial to the conclusion reported by $M M W R$ that human errorthat is, inappropriate connection of the AFER to the bronchoscope by hospital staff-was the cause of the patient injuries described in cluster $3 .^{4}$ If the filtered rinse water had been sampled and found to be contaminated with $P$ aeruginosa (the outbreak microorganism in cluster 3), then the $M M W R$ 's conclusion, which suggests patient-to-patient transmission, would likely be incomplete, and the reported patient injuries might have occurred even if hospital staff had properly connected the bronchoscopes to the AFER.

Although effective and routinely employed by healthcare facilities to reprocess bronchoscopes and gastrointestinal endoscopes, AFERs have their limitations. For example, unlike other processes that use heat, gas, or a plasma to disinfect or sterilize reused instruments, current AFERs immerse the endoscope in a liquid chemical sterilant, requiring that the endoscope be rinsed with a large volume of water to remove potentially toxic chemical residues. This step is arguably the Achilles' heel of AFERs (and other liquidchemical sterilant-based processes), as the success of these devices is therefore vulnerable to, and depends significantly on, the quality of the rinse water, which is difficult to monitor and control. Rinse water that contains microorganisms can recontaminate the endoscope and result in patient infection, even if the cleaning and chemical immersion steps were effective. ${ }^{1-3}$
Only the use of sterile water would virtually eliminate the risk of recontaminating instruments with waterborne microorganisms during rinsing. But using bona fide sterile water for rinsing can be problematic in the clinical setting, and producing it in the healthcare setting is likely to be expensive and impractical and presumably would require periodic microbiological monitoring to ensure the process's effectiveness. In lieu of sterile water, AFERs typically rinse the endoscope with tap water that has been passed through a bacterial filter rated at 0.1 or $0.2 \mu \mathrm{m}$. These filters are designed to produce bacteriafree, but not sterile, water in the healthcare setting. Moreover, these water filters are not fail-safe and, with repeated use, have been reported to fail, allowing microorganisms to pass. $^{1}$

Whereas the contribution of cleaning and disinfecting the endoscope to the prevention of patient infection is well recognized, the importance of drying and properly storing the endoscope is sometimes overlooked. ${ }^{4,5}$ (Whether the hospital involved in cluster 3 dried the endoscope before storage is unclear. ${ }^{5}$ ) Why might a healthcare facility fail to dry its endoscopes before storage? Plausible explanations include misunderstanding the inherent limitations of water filters and confusion over the definitions of, and microbiological differences between, tap water, bottled sterile water, and filtered water claimed to be bacteriafree or sterile. ${ }^{5}$ Using a $70 \%$ alcohol rinse followed by forced air to dry the endoscope before storage is well documented and has been shown to prevent nosocomial infection caused by rinse water contaminated with $P$ aeruginosa, mycobacteria, and other opportunistic pathogens. ${ }^{1,2,5}$ This practice is recommended whether using tap water or water labeled as bacteria-free or sterile. ${ }^{5}$

In conclusion, to prevent patient infection caused by inadequately dried endoscopes, I encourage federal regulatory agencies and professional endoscopy and infection control organizations to reemphasize the importance of thoroughly drying and properly storing the endoscope. Also encouraged, to prevent confusion and patient infection, are discussions aimed at clarifying and detailing the definitions and microbiological dif- 
ferences between the different types of water used to rinse endoscopes. Lacking in the medical literature is a clear description of the parameters of sterile water, how it is produced, and how it compares to, and differs microbiologically from, filtered water claimed to be bacteria-free or sterile. Finally, although the CDC does not recommend routine microbiological sampling of endoscopes or the water used to rinse them, I recommend revisiting the conditions under which such a practice might be indicated to reduce the risk of patient infection.

\section{REFERENCES}

1. Struelens MJ, Rost F, Deplano A, Maas A, Schwan V, Serruys E, et al. Pseudomonas aeruginosa and Enterobacteriaceae bacteremia after biliary endoscopy: an outbreak investigation using DNA macrorestriction analysis. Am J Med 1993;95:489-498.

2. Alvarado CJ, Stolz SM, Maki DG. Nosocomial infections from contaminated endoscopes: a flawed automated endoscope washer. An investigation using molecular epidemiology. Am J Med 1991;91(suppl 3B): 272S-280S.

3. Centers for Disease Control and Prevention.
Nosocomial infection and pseudoinfection from contaminated endoscopes and bronchoscopes-Wisconsin and Missouri. MMWR 1991:40:675-678

4. Centers for Disease Control and Prevention. Bronchoscopy-related infections and pseudoinfections--New York, 1996 and 1998. MMWR 1999;48:557-560.

5. Berlau J. A new risk for high-tech surgery? Investor's Business Daily February 14, 2000;16:A1, A26.

Lawrence F. Muscarella, PhD Custom Ultrasonics, Inc Ivyland, Pennsylvania

\section{Triclosan and Antibiotic Resistance in $S$ aureus}

\section{Gina Pugliese, RN, MS}

Martin S. Favero, PhD

The debate on the connection between chemical germicides and antibiotic resistance continues. Suller and Russell, from the Welsh School of Pharmacy, Cardiff University, recently reported on their studies with triclosan. Triclosan (2,4,4'-trichloro-2'-hydroxydiphenyl ether) is an antimicrobial agent used in hygiene products, plastics, and kitchenware. Handwashing products containing triclosan are used by healthcare workers in wards with patients infected with methicillinresistant Staphylococcus aureus. $S$ aureus strains with low-level resistance to triclosan have emerged. It has been claimed that strains with decreased susceptibility to biocides may also be less susceptible to antibiotics.

They tested the susceptibility of $S$ aureus clinical isolates to triclosan and several antibiotics. Triclosan minimum inhibitory concentrations (MICs) ranged between 0.025 and $1 \mathrm{mg} / \mathrm{L}$. Some, but not all, strains were resistant to several antibiotics and showed low-level triclosan resistance. $S$ aureus mutants with enhanced resistance to triclosan $(<1$ $\mathrm{mg} / \mathrm{L}$ ) were isolated. In several cases this resistance was stably inherited in the absence of triclosan. These mutants were not more resistant than the parent strain to several antibiotics. Changes in triclosan MICs associated with the acquisition of a plasmid encoding mupirocin resistance were not observed, suggesting that the triclosan and mupirocin co-resistance seen in a previous study was not the result of a single resistance gene or separate genes on the same plasmid.

The continuous exposure of a triclosan-sensitive $S$ aureus strain to sub-MIC concentrations of triclosan for 1 month did not result in decreased susceptibility to triclosan or to several antibiotics tested. Triclosan-induced potassium leakage and bactericidal effects on a triclosan-sensitive strain, a resistant strain, and a strain selected for increased resistance were compared with those of non-growing organisms, exponentially growing organisms, and organisms in the stationary phase. No significant differences between the strains were observed under these conditions despite their different MICs.

The authors point out that biocides have multiple target sites, and so MICs often do not correlate with bactericidal activities. The ability of $S$ aureus to develop resistance to triclosan and the current view that triclosan may have a specific target in Escherichia coli, namely enoyl reductase, underline the need for more research on the mechanisms of action and resistance.

FROM: Suller MT, Russell AD. Triclosan and antibiotic resistance in Staphylococcus aureus. I Antimicrob Chemother 2000;46:11-18. 